\title{
Care for chronic renal patients - Role of multidisciplinary education
}

\author{
Á Haris, K Polner \\ Nephrology Department, Szent Margit Hospital, Budapest, Hungary
}

Received: February 27, 2018

Accepted: November 9, 2018

\begin{abstract}
The aim of this study is to summarize the beneficial effects of the holistic approach to patients living with chronic renal failure, including multidisciplinary education and psychosocial care. By education, we enable our patients to reach self-management, appropriate compliance, and coping, which may decrease progression of renal failure, avoid urgent need for starting dialysis and may facilitate better modality selection, access planning, renal transplantation activity, and rehabilitation. Psychosocial care reduces anxiety and fear, modifies perception of illness' burden, and increases quality of life. Yet, both patients and nephrologists feel that transmission of information is often insufficient. Different methods can effectively be utilized as educational interventions, meetings with staff, and also with expert patients, group education sessions, written or online materials, or multimedia presentations. Patient care of such a high complexity can be provided only by multidisciplinary teams. A special Hungarian example of holistic care is presented, and the favorable results of the education and lifestyle camps for patients accompanied by their relatives are discussed. Accordingly, complex care of patients living with chronic renal failure on the long run is cost saving.
\end{abstract}

Keywords: multidisciplinary education, holistic care, psychosocial care, chronic renal failure, quality of life

\section{Introduction}

Chronic renal failure is a lifelong disease that alters almost all aspects of one's daily life. Understanding and accepting the diagnosis and finally learning to live together with this disease need long time, especially for young, previously healthy persons $(1,8)$. Patients, who are compliant and follow medical, dietary, and lifestyle advices, have more favorable outcomes, with prolonged time until renal replacement therapy (RRT) becomes necessary. Educated patients can be rehabilitated more effectively, choose home-based therapies more frequently, and participate in living donor renal transplantation more often $(12,30)$. Nevertheless, developing appropriate attitude and high level of coping with this condition needs capability of handling the emotional stress and following the required lifestyle adjustments and medical restrictions (35).

The major help by a healthcare team can support the persons living with kidney disease and can provide sufficient information and high-quality education $(16,24)$. By education, we enable our patients to reach self-management, to take full responsibility for their own condition, and by having wide range of knowledge that they will be able to appropriately participate in shared decision-making $(5,11)$. However, in order to develop strategies to

Corresponding author: Ágnes Haris, MD, PhD

Nephrology Department, Szent Margit Hospital

132 Bécsi Street, Budapest 1032, Hungary

Phone: +36 30401 1255; Fax: +36 1250 1970; E-mail: agnesharis@hotmail.com 
integrate the disease and its treatment into their daily lives, a lot of specific information of disease are required. Patients need to understand the important aspects of their disease, to monitor symptoms, to learn how to handle smaller problems, and to recognize when and how they should report the relevant ones. They need to stop smoking, optimize physical activity, understand the aim and importance of each of the ordered drugs, also the restricted ones, and how to adjust dosages when an intercurrent disease develops, e.g., decreasing or increasing the dose of diuretics or antihypertensive medications according to the actual condition, or handling the dose of metformin in case of an acute gastrointestinal disease with vomiting and diarrhea. Patients need to be aware of the risks of some over-the-counter medications and herbal drugs. Dietary prescriptions are also not simple, the protein restriction and requirement of low sodium, potassium, and phosphate intake need detailed knowledge of food ingredients, and also need self-discipline. When renal failure progresses and preparation for RRT is unavoidable, patients have to be able to actively participate in modality selection and optimally become wait-listed for renal transplantation $(13,17,28)$.

\section{Gaps in Knowledge of Patients Living with Kidney Disease}

Even if the utmost importance of patient education is well accepted in the nephrological community, we have numerous data about the insufficient transmission of information to our patients (24). Finkelstein et al. (9) studied 676 patients at stage 3-5 chronic kidney disease (CKD) from US and Canada, who attended nephrological clinics for a median of 2 , a mean of 5.2 years, so had multiple chances for discussions about their condition with their healthcare providers. In spite of this, $43 \%$ stated they had no knowledge of hemodialysis (HD), $57 \%$ did not have information about continuous ambulatory peritoneal dialysis (CAPD), $66 \%$ of them did not know automated peritoneal dialysis, and 56\% had no knowledge of transplantation. When the investigators enquired participants about the advantages and disadvantages of various RRT modalities, one third of the patients who had previously indicated perceived knowledge of a certain modality could not provide details of it. As patients from US showed more limited perceived knowledge than subjects from Canada, where many clinics offer multidisciplinary care, investigators reasoned that those who had chance for adequate education for CKD had more information than those who obtained only nephrological care by single nephrologists. Poor understanding of the advantages and risks of renal transplantation by dialyzed patients turned out from a Hungarian study (32). Researchers provided questionnaires to $459 \mathrm{HD}$ patients who are under 70 years of age and asked for participation regardless of eligibility for the transplant procedure. The major interests were the perceptions and attitudes of dialyzed patients toward transplantation. Sociodemographic factors were also collected and analyzed in association with the perceived knowledge. Seventy-one percent of the participants showed keen interest in transplantation. Yet, 25\% reported that they had not heard about transplantation from their doctors, $56 \%$ reported that the received information was insufficient, $35 \%$ believed that transplantation caused more problems than benefits, $46 \%$ had significant fears about the surgery, and $45 \%$ about immunosuppression. Those who wanted to be transplanted were more likely younger ( $51 \pm 11$ vs. $58 \pm 11$ years), men (56\%), employed ( $11 \%$ vs. $4 \%$ ), had higher education, and had prior kidney transplantation ( $15 \% \mathrm{vs.} 7 \%)$. The strongest predictor of positive attitude toward the transplantation was the perception that it is the best modality of RRT. Investigators indicated that comprehensible and evidence-based patient information systems, by facilitating self-management, could enable patients to make fully informed about treatment decisions. 


\section{Information and Educational Requirements of the Patients}

On the other side of the coin are the patients' perceptions of their condition and their significant unmet needs of information about the disease and its treatment both by dialysis and transplantation $(5,13)$. Lewis et al. $(21)$ designed a structured interview research in order to determine the informational needs, problems, and concerns expressed by stage $4 \mathrm{CKD}$ patients. The most significant questions in which the new patients have for CKD clinic were detailed information about their disease, prescribed medications, graft, fistula or catheter insertion, and their care. They also expressed their anxiety about potential financial problems with the forthcoming dialysis treatment, and enquired about dietary restrictions and how they can maintain former social life, personal relationships, and vacations. Importantly, they had lots of concerns about initiation of RRT. Patients also pointed out some of the insufficiently discussed issues, such as sexual life with renal failure (27).

\section{Aims and Results of Patients' Education}

Several high-quality investigations showed beneficiary effects of the multidisciplinary patient care $(3,12,30,34)$. One of the landmark studies completed by Curtis et al. (7) highlighted the long-term benefits of patient's education. In this retrospective observational investigation, performed in Canada and Italy, 288 patients participated. They attended either multidisciplinary or standard nephrological care clinics for an average of 42, but at least 3 months before dialysis initiation. Survival was evaluated after initiating RRT. The multidisciplinary care provider team consisted of a nephrologist, a nurse educator, a social worker, a dietician, a pharmacist, and a psychologist. Each educational session lasted for $1.5 \mathrm{~h}$ per visit, and approximately 5 visits/year were scheduled for everyone. Subjects who obtained standard nephrological care for approximately $0.5 \mathrm{~h}$ per visit served as controls. It is important to point out that all of these patients were followed by renal service, and no unplanned dialysis initiation occurred because of late referral. Patients were followed for a median of 14 months after dialysis initiation. Kaplan-Meier survival analysis showed that those who had been exposed to multidisciplinary education prior to dialysis initiation had significantly longer survival compared to the regularly cared controls. According to multivariate Cox proportional hazards modeling, besides age, the exposure to multidisciplinary education was the only significant predictor of survival [hazard ratio for death was 2.17 (95\% CI: 1.11-4.28) for those who achieved standard vs. multidisciplinary care].

The effect of multidisciplinary predialysis education was evaluated by Wu et al. (37) in a prospective observational study conducted in Taiwan. Fifty percent, as much as 287 subjects among all CKD patients at stage 3-5 who had been referred to nephrology during the time frame of the investigation attended multidisciplinary predialysis educational sessions. Those who obtained standard nephrological care served as controls. The educational program followed the National Kidney Foundation Dialysis Outcomes Quality Initiative, and was adjusted to the CKD stage. During the 12-month follow-up, progression of renal failure and mortality decreased significantly, and the dialysis initiation and hospitalization rate were also reduced in the multidisciplinary educated patients than in controls [changes of glomerular filtration rate (GFR) were $0.008 \pm 0.139$ vs. $-0.113 \pm 0.786 \mathrm{ml} / \mathrm{min} / \mathrm{month}$, mortality $1.7 \%$ vs. $10 \%$, dialysis initiation $14 \%$ vs. $43 \%$, 1 -year hospitalization rate $2.8 \%$ vs. $16.4 \%$ in the multidisciplinary educated patients vs. controls]. Limited patients underwent HD with temporary vascular catheter in the educated group compared to controls ( $25 \%$ vs. $50.4 \%$ ), 
and peritoneal dialysis (PD) was selected as choice of modality by $35 \%$ versus $20.5 \%$ of patients in the groups, respectively.

In a Spanish study, Marrón et al. (23) investigated the consequences of education on dialysis modality selection. Only half of the 626 patients who started RRT had been educated on dialysis modalities during this study (2002). Educated patients selected PD in $31 \%$, whereas non-educated patients only in $8.3 \%$. Dialysis education was associated with a planned RRT start in $73.4 \%$, but among non-educated patients it occurred only in $26.6 \%$.

The effectiveness of education on modality selection and dialysis access planning was also demonstrated by the results of the Nationwide Predialysis Educational Program [treatment options programs (TOPs)], organized by Fresenius Medical Care, North America (19). Between 2006 and 2008, more than 20,000 patients attended classes, and $28 \%$ of them started dialysis. Twenty-five percent of TOPs-educated patients began home-based RRTs, whereas among non-TOPs-educated subjects, only 3.3\% chose home modalities. Outcome data of a cohort of 2,800 TOPs participants were compared to a matched cohort of 2,800 nonTOPs patients. The case-mix plus laboratory's adjusted odds ratio of choosing PD versus HD was 7.73 [95\% CI: 3.26-18.32], odds ratio of having a fistula or graft at the initiation of HD was 1.73 [95\% CI: $1.51-1.97$ ], the hazard ratio for death was 0.61 [95\% CI: $0.50-0.74$ ] for TOPs attendees versus non-TOPs participants.

In order to develop highly effective educational intervention to initiate self-care dialysis, Manns et al. (22) initially provided written materials and presented a short video about different self-care renal replacement modalities to their previously multidisciplinary educated patients. In the second phase of this small randomized controlled study, problem-solving interactive sessions about home-based dialysis options were organized for small groups of patients. The investigators found significant improvements of their patients' knowledge and attitudes toward self-care dialysis compared to those who did not participate in such an education. The most effective educational form was the small-group interactive session.

In another type of personalized educational program, self-management patient support was provided (2). In this open-label randomized controlled trial of stage 3-5 CKD patients, besides standardized multidisciplinary education, weekly telephone-based supports were provided to help patients with self-management. Investigators found decreased rate of disease progression and hospitalization, explained these results by better adherence, compliance, self-management, and improved relationship between patients and clinicians.

Although the aforementioned investigations impressively showed the beneficial effects of the multidisciplinary education, it has to be pointed out that patient's selection for observational studies could be potentially biased, as subjects with higher education, less comorbidities, or better social support have higher chance to be involved in such educational activities. This might largely influence the results, which is a major limitation of these studies. Unfortunately, even the investigations with random selection of patient lacked proper study design due to insufficient standard patient care for controls, or lack of long-term follow-up.

In addition to the sophisticated educational interventions, even simple tools can increase our patients' knowledge. The impact of physician-delivered educational interventions was studied by distributing kidney education worksheets, aiming facilitated discussions during the forthcoming nephrology visits between patients and the attending nephrologists (36). These two sided, easy to administered cards summarized the most important basic information about the disease and strategies for its care, using easily understandable language and simplified graphics. This time-efficient educational tool was found to be beneficial both by patients and investigators to increase the awareness and knowledge of the disease. 
Realizing the above-discussed difficulties and the utmost importance of patient's education, a consensus conference was held in 2013 and published by Isnard Bagnis et al. (14). Accordingly, the most important benefits of patient education are the reduced urgent start of dialysis, decreased rate of hospitalization, earlier, timely placement of vascular access/PD catheter, greater likelihood of choosing self-modality, extended time until dialysis becomes necessary, better compliance, reduced anxiety and fear, and decreased mortality. The educational team should consist of nephrologists and CKD nurses at a minimum, but ideally comprises a multidisciplinary team with a nephrologist, a CKD nurse, a dietician, a social worker, a mental health professional, a physical therapist, and expert patients who are willing to demonstrate their own difficulties, experiences, and personal views. Suggested teaching materials can be provided by one-to-one meetings with the staff, but also on group education sessions, by written booklets at an appropriate level to disease stage and to patient's level of education, paying attention to the cultural-religious background as well. Multimedia presentations can also be used effectively, and also online materials, but these have to be used on carefully selected websites. Brief tours in dialysis facilities are highly suggested, and meetings or videos with expert patients are also useful. Even if education is time-consuming, the aforementioned benefits provide cost savings on the long run $(4,20)$.

The European Renal Best Practice Advisory Board issued clinical advices, which state that the initial modality choice should primarily be made by the sufficiently informed patients who have been provided well-balanced information about renal replacement modalities by means of structured education (6). Similarly, detailed information is necessary for shared decision-making in initiation or withdrawal of dialysis (10).

\section{Role of the Psychosocial Care}

Besides education, psychosocial support is a cornerstone of the complex, holistic care of patients suffering from lifelong diseases $(27,31)$. Most of the chronic disorders lead to a gradually deteriorating physical condition, which hampers both daily activities and work. Renal patients often lose their jobs, then financial difficulties evolve, which result in depression and fear. Psychosocial care may substantially modify the patient's perception of well-being and perception of burden of illness, and may significantly affect quality of life (35). It is important to remember that patient's assessment, how the disease interferes with his/her life influences their ability to cope with the altered condition, and this determines compliance, which, on the long run, affects survival $(8,25)$. In case of non-compliance, rate of hospitalization and mortality increases, and the rehabilitation potential diminishes.

The close association of psychosocial factors and non-compliance in HD and peritoneal dialysis patients was demonstrated by the study of Kutner et al. (18). Decreased perceived self-control over future health and perceived negative effects of kidney disease on daily life predicted non-compliance with dialysis prescriptions, including skipped or shortened HD sessions, missed PD exchanges, or high serum phosphate levels, which occurred as frequently as in one third of patients. On the other hand, Kimmel et al. (15) demonstrated that higher levels of perceived social support, improved perception of the effect of illness, and increased behavioral compliance were associated with decreased mortality risk by $20 \%-23 \%$ in 295 HD patients, followed for $26.4 \pm 12.8$ months. Perceived psychosocial support and participation in shared decision-making increase the patients' satisfaction with their dialysis treatment (26), and also their decisions about being wait-listed for renal transplantation (33). Others found inverse relationship between social support and depression in HD patients (29). 


\section{Education and Psychosocial Care of Patients and their Relatives - The Special Hungarian Experience}

In 2007, the renal team of Szent Margit Hospital, Budapest, initiated to organize education and lifestyle camps for patients and their relatives (Fig. 1). For these camps, the registration for patients is voluntary; the only "requirement" is bringing one of the relatives. All the expenses are covered by our Szent Margit Hospital Kidney Foundation, completely aiming free participation for everyone. The education lasts for a whole week. Groups, each with 20-25 participants, have been mainly organized for predialysis patients with CKD 3-5 and in few occasions for peritoneal dialysis and HD patients as well. Between 2007 and 2017, as much as 14 groups were organized, in which 151 patients and 154 relatives participated.

In these camp sessions of multidisciplinary education, we cover all important topics a renal patient and his/her relative ideally should know. These are the basic functions of the kidney, fluid, and electrolyte balance; significance of the laboratory results; and the outstanding importance of diet and drug treatments. We discuss RRT modalities, living and cadaver donor kidney transplantation, major comorbidities, and also the options to prevent and treat them. We provide presentations regarding assistance of psychosocial problems, chances for rehabilitation, legal rights of the patients, and how we can alleviate their handicaps. The educational team comprises nephrologists, educational nurses, dieticians, social workers, psychologists, rehabilitation specialists, and physiotherapists. Since 2010, a Tai-chi educator has joined the team, organizes daily activities, which result in relaxation and great pleasure of the participants. Education is completed by voluntary case presentations of patients who have already experienced some mile stones of the troublesome life of renal patients, to transfer personal experiences, e.g., how the most suitable RRT modality can be chosen; what kind of advantages and risks of kidney transplantation may be expected; and how to fight, handle, and accept this chronic disease. Voluntary relatives speak about their commitment for kidney donations, and their recipients may present how to become willing to accept these offerings. Collective discussions of educative videos and difficult cases are also parts of the sessions.

The whole-week-long duration provides the opportunity of extensive teaching and learning. Patients are very enthusiastic, and by involving the relatives, the learning process becomes even more effective and the personal activity is further enhanced. Along with these sessions, we have also experienced that the accompanying relatives gradually realized how difficult the patients' lives are and how they can support them. When the entire family fights together, the patients accept their disease more easily. We put great emphasis on psychoeducation and self-knowledge, aiming to strengthen the participants' psychological balance, helping them to accept their disease, to develop coping strategies, and to escape stress and
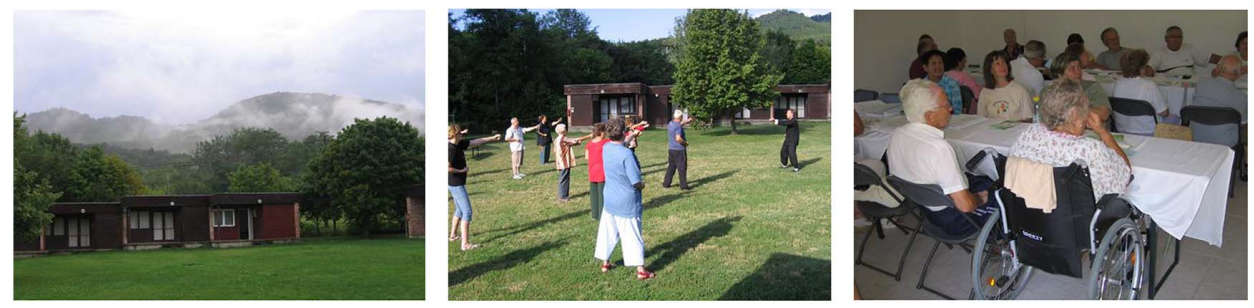

Fig. 1. Daily activities in education and lifestyle camps for patients and their relatives the Hungarian experience 
depression. We discuss chapters of two books, Living with kidney disease by Kerstin Bergström and Per Ake Zillén, who put great emphasis on self-management based on own experiences, and Life Lessons by Elizabeth Kübler-Ross and David Kessler, which teaches how to live high-quality happy life for those who suffer from severe diseases.

After each camp, we found better blood pressure results, improving phosphate balance, dietary, and drug compliance. The critical long-term benefit has been shown by significantly increased home-based RRT modality selection, and also by increased activity for being waitlisted for kidney transplantation. Moreover, a higher number of kidney donations have been offered by healthy relatives. Taking together our experiences, these camps with complex, holistic view have proved to be a very effective multidisciplinary educational modality both for our patients and their relatives, which resulted in outstanding outcome, delayed initiation of dialysis, and improved quality of life. Meanwhile, a respectful partnership has been developed between healthcare providers and patients. The history of one of our patients, presented briefly in Appendix 1 and Fig. 2, demonstrates the influence of this holistic care on his behavioral change and improved coping.

It has to be mentioned that patient selection for our observational study might be biased by selecting educated, compliant patients. We invited those people who showed good attitude toward education. All participants had been accompanied by their family members, which referred to substantial psychosocial support. In spite of these shortcomings, we felt important to present our experiences with such an educational modality, which has been proven to be a very effective learning experience for patients and their relatives as well.

\section{Summary}

Multidisciplinary education of patients living with chronic renal failure improves several aspects of outcome measures, such as reduced urgent start of dialysis, prolongation of predialysis period, enhancement of home-based RRT selection, and reduced hospitalization

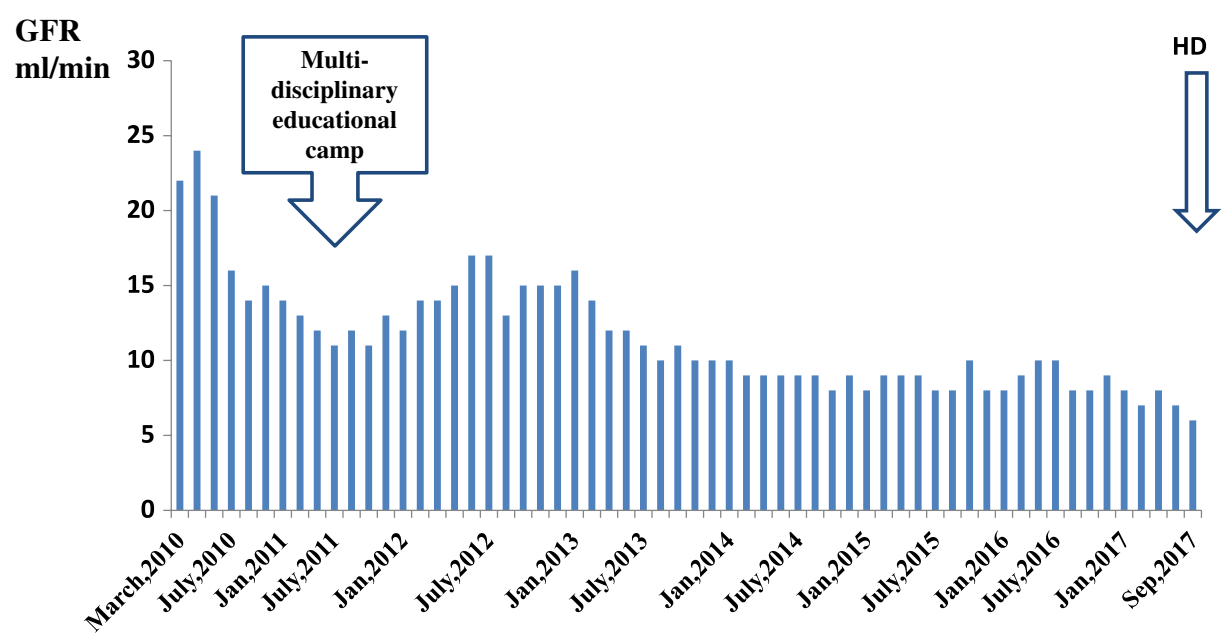

Fig. 2. Graph shows GFR values before and after multidisciplinary education of one of the patients who participated in the education and lifestyle camp organized by Szent Margit Hospital Kidney Foundation (brief history of the patient is summarized in Appendix 1) 
and mortality. Implementing holistic view with complex medical and psychosocial care and involving our patients' relatives result in more effective education, achieve improved coping and adherence, reduce anxiety, and provide better chance for rehabilitation. Although the complex care of renal patients is time-consuming and needs multiple efforts of the renal team, on the long-term, this results in cost savings. The concept and aim of holistic care is the commitment of the 2012 Budapest Declaration of the International Federation of Kidney Foundations (IFKF), as “.... develop and implement, as a priority, comprehensive programs for the screening, prevention, treatment and rehabilitation of individuals living with chronic kidney disease" and "... in a holistic manner, to achieve the best health outcomes, including rehabilitation and quality of life" (Appendix 2).

\section{REFERENCES}

1. Chan R: The effect of acceptance on health outcomes in patients with chronic kidney disease. Nephrol. Dial. Transplant. 28, 11-14 (2013)

2. Chen SH, Tsai YF, Sun CY, Wu IW, Lee CC, Wu MS: The impact of self-management support on the progression of chronic kidney disease - a prospective randomized controlled trial. Nephrol. Dial. Transplant. 26, 3560-3566 (2011)

3. Chen YR, Yang Y, Wang SC, Chiu PF, Chou WY, Lin CY, Chang JM, Chen TW, Ferng SH, Lin CL: Effectiveness of multidisciplinary care for chronic kidney disease in Taiwan: a 3-year prospective cohort study. Nephrol. Dial. Transplant. 28, 671-682 (2013)

4. Chen YR, Yang Y, Wang SC, Chou WY, Chiu PF, Lin CY, Tsai WC, Chang JM, Chen TW, Ferng SH, Lin CL: Multidisciplinary care improves clinical outcome and reduces medical costs for pre-end-stage renal disease in Taiwan. Nephrology (Carlton). 19, 699-707 (2014)

5. Costantini L, Beanlands H, McCay E, Cattran D, Hladunewich M, Francis D: The self-management experience of people with mild to moderate chronic kidney disease. Nephrol. Nurs. J. 35, 147-155, quiz 156 (2008)

6. Covic A, Bammens B, Lobbedez T, Segall L, Heimbürger O, van Biesen W, Fouque D, Vanholder R: Educating end-stage renal disease patients on dialysis modality selection. NDT Plus 3, 225-233 (2010)

7. Curtis BM, Ravani P, Malberti F, Kennett F, Taylor PA, Djurdjev O, Levin A: The short- and long-term impact of multi-disciplinary clinics in addition to standard nephrology care on patient outcomes. Nephrol. Dial. Transplant. 20, 147-154 (2005)

8. Curtis CE, Rothstein M, Hong BA: Stage-specific educational interventions for patients with end-stage renal disease: psychological and psychiatric considerations. Prog. Transplant. 19, 18-24 (2009)

9. Finkelstein FO, Story K, Firanek C, Barre P, Takano T, Soroka S, Mujais S, Rodd K, Mendelssohn D: Perceived knowledge among patients cared for by nephrologists about chronic kidney disease and end-stage renal disease therapies. Kidney Int. 74, 1178-1184 (2008)

10. Galla JH: Clinical practice guideline on shared decision-making in the appropriate initiation of and withdrawal from dialysis. The Renal Physicians Association and the American Society of Nephrology. J. Am. Soc. Nephrol. 11, 1340-1342 (2000)

11. Golper T: Patient education: can it maximize the success of therapy? Nephrol. Dial. Transplant. 16, 20-24 (2001)

12. Goovaerts T, Jadoul M, Goffin E: Influence of a Pre-Dialysis Education Programme (PDEP) on the mode of renal replacement therapy. Nephrol. Dial. Transplant. 20, 1842-1847 (2005)

13. Huang X, Carrero JJ: Better prevention than cure: optimal patient preparation for renal replacement therapy. Kidney Int. 85, 507-510 (2014)

14. Isnard Bagnis C, Crepaldi C, Dean J, Goovaerts T, Melander S, Nilsson EL, Prieto-Velasco M, Trujillo C, Zambon R, Mooney A: Quality standards for predialysis education: results from a consensus conference. Nephrol. Dial. Transplant. 30, 1058-1066 (2015)

15. Kimmel PL, Peterson RA, Weihs KL, Simmens SJ, Alleyne S, Cruz I, Veis JH: Psychosocial factors, behavioral compliance and survival in urban hemodialysis patients. Kidney Int. 54, 245-254 (1998)

16. Kulcsar I: How can we improve the chronic renal patient care? Hypertonia és Nephrologia 21, 73-78 (2017)

17. Kurella Tamura M, Li S, Chen SC, Cavanaugh KL, Whaley-Connell AT, McCullough PA, Mehrota RL: Educational programs improve the preparation for dialysis and survival of patients with chronic kidney disease. Kidney Int. 85, 686-692 (2014) 
18. Kutner NG, Zhang R, McClellan WM, Cole SA: Psychosocial predictors of non-compliance in haemodialysis and peritoneal dialysis patients. Nephrol. Dial. Transplant. 17, 93-99 (2002)

19. Lacson E, Jr., Wang W, DeVries C, Leste K, Hakim RM, Lazarus M, Pulliam J: Effects of a nationwide predialysis educational program on modality choice, vascular access, and patient outcomes. Am. J. Kidney Dis. 58, 235-242 (2011)

20. Lei CC, Lee PH, Hsu YC, Chang HY, Tung CW, Shih YH, Lin CL: Educational intervention in CKD retards disease progression and reduces medical costs for patients with stage 5 CKD. Ren. Fail. 35, 9-16 (2013)

21. Lewis AL, Stabler KA, Welch JL: Perceived informational needs, problems, or concerns among patients with stage 4 chronic kidney disease. Nephrol. Nurs. J. 37, 143-148, quiz 149 (2010)

22. Manns BJ, Taub K, VanderStraeten C, Jones H, Mills C, Visser M, McLaughlin K: The impact of education on chronic kidney disease patients' plans to initiate dialysis with self-care dialysis: a randomized trial. Kidney Int. 68, 1777-1783 (2005)

23. Marrón B, Martinez Ocana JC, Salgueira M, Barril G, Lamas JM, Martin M, Sierra T, Rodriguez-Carmona A, Soldevilla A, Martinez F: Impact of end-stage renal disease care in planned dialysis start and type of renal replacement therapy - a Spanish multicentre experience. Nephrol. Dial. Transplant. 21, ii51-ii55 (2006)

24. Narva AS, Norton JM, Boulware LE: Educating patients about CKD: the path to self-management and patientcentered care. Clin. J. Am. Soc. Nephrol. 11, 694-703 (2016)

25. Parfeni M, Nistor I, Covic A: A systematic review regarding the association of illness perception and survival among end-stage renal disease patients. Nephrol. Dial. Transplant. 28, 2407-2414 (2013)

26. Robinski M, Mau W, Wienke A, Girndt M: The Choice of Renal Replacement Therapy (CORETH) project: dialysis patients' psychosocial characteristics and treatment satisfaction. Nephrol. Dial. Transplant. 32, 315-324 (2017)

27. Schipper K, Abma TA: Coping, family and mastery: top priorities for social science research by patients with chronic kidney disease. Nephrol. Dial. Transplant. 26, 3189-3195 (2011)

28. Song MK, Lin FC, Gilet CA, Arnold RM, Bridgman JC, Ward SE: Patient perspectives on informed decisionmaking surrounding dialysis initiation. Nephrol. Dial. Transplant. 28, 2815-2823 (2013)

29. Tezel A, Karabulutlu E, Sahin Ö: Depression and perceived social support from family in Turkish patients with chronic renal failure treated by hemodialysis. J. Res. Med. Sci. 16, 666-673 (2011)

30. Thanamayooran S, Rose C, Hirsch DJ: Effectiveness of a multidisciplinary kidney disease clinic in achieving treatment guideline targets. Nephrol. Dial. Transplant. 20, 2385-2393 (2005)

31. Thong MSY, Kaptein AA, Krediet RT, Boeschoten EW, Dekker FW: Social support predicts survival in dialysis patients. Nephrol. Dial. Transplant. 22, 845-850 (2007)

32. Vamos EP, Csepanyi G, Zambo M, Molnar MZs, Rethelyi J, Kovacs A, Marton A, Nemeth Zs, Novak M, Mucsi I: Sociodemographic factors and patient perceptions are associated with attitudes to kidney transplantation among haemodialysis patients. Nephrol. Dial. Transplant. 24, 653-660 (2009)

33. Vamos EP, Novak M, Mucsi I: Non-medical factors influencing access to renal transplantation. Int. Urol. Nephrol. 41, 607-616 (2009)

34. Wang SM, Hsiao LC, Ting IW, Yu TM, Liang CC, Kuo HL, Chang CT, Liu JH, Chou CY, Huang CC: Multidisciplinary care in patients with chronic kidney disease: a systematic review and meta-analysis. Eur. J. Intern. Med. 26, 640-645 (2015)

35. White C, McDonnell H: Psychosocial distress in patients with end-stage kidney disease. J. Ren. Care. 40, 74-81 (2014)

36. Wright Nunes J, Greene J, Wallston K, Eden S, Shintani A, Elasy T, Rothman R, Ikizler TA, Cavanaugh K: Pilot study of a physician-delivered education tool to increase patient knowledge about CKD. Am. J. Kidney Dis. 62, 23-32 (2013)

37. Wu IW, Wang SY, Hsu KH, Lee CC, Sun CY, Tsai CJ, Wu MS: Multidisciplinary predialysis education decreases the incidence of dialysis and reduces mortality - a controlled cohort study based on the NKF/DOQI guidelines. Nephrol. Dial. Transplant. 24, 3426-3433 (2009) 


\section{APPENDIX 1}

The 61-year-old male patient was diagnosed with type-II diabetes and hypertension in 1989. In 1998, insulin administration had to be started, but the patient did not maintain diabetic diet, and his blood sugar level was permanently unsatisfactory. Unfortunately, his wife did not believe in the importance of the diet either, and did not support him. In 2007, proteinuria and increased serum creatinine $(143 \mu \mathrm{mol} / \mathrm{L})$ were detected. He obtained nephrological care in our hospital in 2009, by that time, GFR was changed to $21 \mathrm{ml} / \mathrm{min}$ and serum creatinine to $296 \mu \mathrm{mol} / \mathrm{L}$. At that time, he still refused diet, and took his medications irregularly. In 2011, serum creatinine increased to $546 \mu \mathrm{mol} / \mathrm{L}$, GFR decreased to $11 \mathrm{ml} / \mathrm{min}$, and the attending nephrologist suggested arteriovenous (AV) fistula creation and HD planning. But in July 2011, he was successfully convinced to register in the education and lifestyle camp organized by Szent Margit Hospital Kidney Foundation. After participation and completion of this multidisciplinary education, his and outstandingly his wife's behavior completely changed. They understood the importance of diet and other requirements and documented dietary diary together. The patient started following dietary restrictions, stopped smoking, monitored his blood pressure, and took his medications regularly. His wife also realized the importance of following medical advice, and since that time she has become a firm support of her husband. Not only has she cared for dietary prescriptions, helped measuring blood sugar and blood pressure, but the couple's close emotional partnership has increased the patient's ability for high level of coping, accepting his disease, being compliant with the prescriptions, and making appropriate decisions regarding his own health. Dialysis initiation could be delayed more than 6 years, and had to be started only in September 2017. At present, the patient is in good physical condition and $\mathrm{HD}$ is performed thrice weekly via a properly functioning AV fistula (CAPD could not be initiated, because of severe visual deficiency). Graph shows his GFR values before multidisciplinary education and after the camp (Fig. 2). 


\section{APPENDIX 2}

\section{The 2012 Budapest Declaration of the IFKF A holistic approach to address the bio-psycho-socio-spiritual needs of individuals living with chronic kidney disease}

Chronic Kidney Disease poses a growing concern for society in both developed and developing countries. It is the responsibility of the medical and health care communities, governments and civil bodies to act collaboratively to address the challenges for patients living with chronic kidney disease in a holistic manner, to achieve the best health outcomes, including rehabilitation and quality of life.

We recommend that national governments in collaboration with international and nongovernmental organizations develop and implement, as a priority, comprehensive programs for the screening, prevention, treatment and rehabilitation of individuals living with chronic kidney disease.

We recommend that these organizations increase the level of their funding for the advancement of clinical and basic scientific research related to kidney disease of all types with the ultimate goal of ensuring a continual provision and application of the most advanced knowledge and technical advances for the benefit of kidney patients. The translation of scientific research findings into clinical practice should be facilitated and supported.

We recommend that medical communities employ a holistic approach for the treatment of patients living with chronic kidney disease, recognizing all their bio-psycho-socio-spiritual and somatic needs. It is important to combine the cutting-edge modern technology of the $21^{\text {st }}$ century with the methods of healing focusing on the whole person and individual care to ensure better efficacy in the prevention, treatment and rehabilitation.

Educational activities of scientists, healthcare professionals, kidney patients and the public-atlarge regarding prevention, detection and treatment of kidney disease in all its aspects should be recognized and supported. These goals should become a priority and focus for governments and non-governmental organizations. Education, as an essential step in the self-care by patients, has received less attention and deserves more focus and support.

We believe that implementing these recommendations and actions will have a substantial effect on the outcomes and quality of life for our patients and will contribute to the sustainable development of medicine and mankind as a whole.

Budapest, 25/08/2012

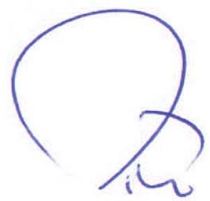

László Rosivall

President of the $13^{\text {th }}$ IFKF AnnualMeeting
Signed and endorsed by:

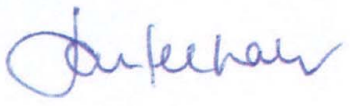

John Feehally

President of ISN
Timur Erk

President of IFKF 